\title{
Yes-associated protein regulates the growth of human non-small cell lung cancer in response to matrix stiffness
}

\author{
YONGGANG YUAN $^{1,2}$, WEILIANG ZHONG ${ }^{3}$, GE MA $^{2}$, BAOXIANG ZHANG $^{4}$ and HUI TIAN ${ }^{1}$ \\ ${ }^{1}$ Department of Thoracic Surgery, Qi Lu Hospital, Shandong Medical University, Jinan, Shandong 250100; ${ }^{2}$ Department of \\ Thoracic Surgery, Yidu Central Hospital, Weifang Medical University, Weifang, Shandong 262500; ${ }^{3}$ Department of \\ Orthopedics, First Affiliated Hospital of Dalian Medical University, Dalian, Liaoning 116011; ${ }^{4}$ Department of \\ Dermatology, Yidu Central Hospital, Weifang Medical University, Weifang, Shandong 262500, P.R. China
}

Received April 8, 2014; Accepted July 22, 2014

DOI: $10.3892 / \mathrm{mmr} .2015 .3231$

\begin{abstract}
The Yes-associated protein (YAP) transcriptional coactivator is recognized as a crucial regulator of human cancer. However, its involvement in human non-small cell lung cancer (NSCLC) in response to physical cues remains unclear. In this study, substrates with different rigidity were generated in order to evaluate the role of YAP, and its upstream regulators in the Hippo pathway, in the regulation of growth of an NSCLC cell line within particular environments. It was shown that the expression of the YAP protein in SPCA-1 NSCLC cells was significantly increased when cultured on a stiff substrate compared to a soft substrate. However, the expression of phospho-YAP protein and large tumor suppressor kinase 1 (LATS1) were markedly decreased after culturing on the stiff substrate. Phosphorylation of YAP by LATS1 leads to cytoplasmic retention of YAP, which inhibits its function as a nuclear transcription coactivator. The study also found that the stiff substrate promoted the growth of NSCLC cells in vitro, and an increase in the transcription levels of Survivin, connective tissue growth factor, amphiregulin and Ki67, as well as a decrease in the expression level of YAP in the cytoplasm, and adecrease in p-YAP. In conclusion, the findings showed that the stiffness of the subcellular matrix altered the behavior of NSCLC cells, and that YAP regulated the growth of NSCLC cells in response to matrix stiffness, thereby suggesting a role for the Hippo-YAP pathway in the response of NSCLC cell growth to specific microenvironments.
\end{abstract}

Correspondence to: Professor Hui Tian, Department of Thoracic Surgery, Qi Lu Hospital, Shandong Medical University, 27 South Shanda Road, Jinan, Shandong 250100, P.R. China

E-mail: wlzhong2012@gmail.com

Key words: Yes-associated protein, non-small cell lung cancer, substrate stiffness, proliferation, Hippo pathway

\section{Introduction}

Lung cancer is one of the leading causes of cancer-related mortality (1). Non-small cell lung cancer (NSCLC) accounts for up to $85 \%$ of lung cancer cases and is usually resistant to chemotherapy (2). Cancer cells in the lung experience mechanical forces associated with normal breathing as well as altered mechanical environments due to pre-existing lung disease or tumor-associated changes. Recent studies have suggested that the mechanical environment of cancerous tumors is involved in tumor formation and metastasis $(3,4)$.

Extracellular matrix (ECM) rigidity, which provides biophysical cues from the microenvironment, has been shown to have an effect on a number of aspects of tumor cell behavior, including adhesion, migration, proliferation and invasion. When cells undergo transformation and initiate the formation of a solid tumor mass, they cause profound changes in the phenotypes of the cells that surround them (5). It has been demonstrated that the majority of solid tumors have increased ECM stiffness, which may lead to increased activation of protumor signaling pathways, such as focal adhesion kinase (FAK) and Ras homolog gene family, member A (RhoA) pathways $(6,7)$. The transcription coactivator Yes-associated protein (YAP) is an important downstream effector of the Hippo pathway (8). Investigation into the role of YAP is a burgeoning field of research, with the focus on transcriptional regulation of a number of aspects of cell behavior. It has been shown that YAP and transcriptional coactivator with PDZ-binding motif (TAZ) are regulators of the nuclear transduction of physical cues in mesenchymal stem cells $(9,10)$ and that increased matrix stiffness may also result in increased activity of the oncogenic YAP/TAZ complex (11). It is therefore evident that NSCLC may develop in an environment with mechanical properties distinct from those encountered in the normal lung.

Despite the growing evidence suggesting that YAP is a crucial regulator of human cancer (11), its involvement in the development of NSCLC in response to mechanical cues remains unclear. In the present study, substrates with different rigidity were generated in order to evaluate the role of YAP, and its upstream regulators in the Hippo pathway, in the regulation of NSCLC cell growth. It was hypothesized that YAP 
Table I. Primer sequences used for RT-qPCR.

\begin{tabular}{|c|c|c|}
\hline Gene & PCR primer sequences (forward and reverse) & GenBank accession no. \\
\hline \multirow[t]{2}{*}{ Survivin } & Forward: 5'-AGGACCACCGCATCTCTACAT-3' & NM_001012270 \\
\hline & Reverse: 5'-AAGTCTGGCTCGTTCTCAGTG-3' & \\
\hline \multirow[t]{2}{*}{ CTGF } & Forward: 5'-ACCGACTGGAAGACACGTTTG-3' & NM_001901 \\
\hline & Reverse: 5'-CCAGGTCAGCTTCGCAAGG-3' & \\
\hline \multirow[t]{2}{*}{ AREG } & Forward: 5'-CTGGGAAGCGTGAACCATTTT-3' & NM_001657 \\
\hline & Reverse: 5'-TCTGAGTAGTCATAGTCGGCTC-3' & \\
\hline \multirow{2}{*}{ Ki67 } & Forward: 5'-ACGAGACGCCTGGTTACTATC-3' & NM 002417 \\
\hline & Reverse: 5'-GCTCATCAATAACAGACCCATTTAC-3' & \\
\hline \multirow{2}{*}{$\beta$-actin } & Forward: 5'-CATGTACGTTGCTATCCAGGC-3' & NM_001101 \\
\hline & Reverse: 5'-CTCCTTAATGTCACGCACGAT-3' & \\
\hline
\end{tabular}

CTGF, connective tissue growth factor; AREG, amphiregulin; RT-qPCR, reverse transcription-quantitative polymerase chain reaction.

may be a bridging molecule between NSCLC growth and the mechanical microenvironment. The findings demonstrate that YAP may regulate the growth of NSCLC in response to matrix stiffness, thereby suggesting a role of the Hippo-YAP pathway in NSCLC growth in response to its physical microenvironment.

\section{Materials and methods}

Generation of substrates with different rigidity. Substrates with modifiable mechanical properties were generated on variably compliant polyacrylamide (PA) gels, according to a previously established method (12). Briefly, glass slides were treated with 3-aminopropyltrimethoxysilane and $0.5 \%$ glutaraldehyde. PA gel solutions with the desired concentrations of acrylamide and bis-acrylamide were allowed to polymerize. The elastic modulus of the PA gel substrates was controlled by adjusting the quantity of acrylamide and bis-acrylamide crosslinker, as previously described (13). Sulfo-SANPAH (Proteochem, Denver, CO, USA) was used to link fibronectin $(100 \mu \mathrm{g} / \mathrm{ml}$; Sigma, St. Louis, MO, USA) to the PA gel surface. The PA gel modulus of elasticity was quantified using atomic force microscopy (AFM, Bruker, Billerica, MA, USA). All substrates were sterilized using UV light.

Cell culture. The SPCA-1 human non-small cell lung cancer cell line was purchased from the American Type Culture Collection (Manassas, VA, USA) and maintained in RPMI-1640 medium (Sigma-Aldrich, St. Louis, MO, USA), supplemented with $10 \%$ fetal bovine serum, $100 \mathrm{U} / \mathrm{ml}$ penicillin, and $100 \mathrm{U} / \mathrm{ml}$ streptomycin (Sigma-Aldrich) at $37^{\circ} \mathrm{C}$ in $5 \% \mathrm{CO}_{2}$ and $95 \%$ relative humidity. The cells were then cultured on PA substrates for up to $48 \mathrm{~h}$ (Fig. 1A). A density of $1 \times 10^{5}$ cells $/ \mathrm{cm}^{2}$ was used to seed the substrates. The proliferation rates were determined by quantifying the cells using a hemocytometer subsequent to trypsinization in each condition. Growth curves for the cells were then determined.

Small-interfering RNA (siRNA) transfection of YAP. siRNAs (siControl and siYAP) specific for the target genes were purchased from Santa Cruz Biotechnology, Inc. (Santa Cruz, CA, USA). For the knockdown of YAP, the corresponding siRNAs were delivered into SPCA-1 cells using Lipofectamine ${ }^{\circledR}$ RNAiMAX (Invitrogen Life Technologies, Carlsbad, CA, USA) according to the manufacturer's instructions.

Immunofluorescence staining. Samples were fixed with $4 \%$ paraformaldehyde for $15 \mathrm{~min}$ and then permeabilized with $0.1 \%$ Triton X-100 for $10 \mathrm{~min}$. After washing with phosphate-buffered saline (PBS) three times, the samples were blocked with normal goat serum (Invitrogen Life Technologies) for $30 \mathrm{~min}$ to prevent nonspecific binding, incubated with polyclonal rabbit primary antibodies against YAP (sc-15407; Santa Cruz Biotechnology, Inc.) at $4^{\circ} \mathrm{C}$ overnight and then incubated with goat anti-rabbit IgG fluorescein isothiocyanate (FITC)-conjugated secondary antibodies (sc-2012; Santa Cruz Biotechnology, Inc.) for $1 \mathrm{~h}$. After staining, the samples were washed with PBS two to three times and imaged using fluorescence microscopy (Olympus IX71; Olympus, Tokyo, Japan). The percentage of cells with YAP expression localized to the nucleus (nuclear YAP) in 10 different visual fields for three replicates. As in the method described previously (14), distribution patterns of Yap expression in the cells were classified as nuclear (nuclear expression $>$ cytoplasmic expression), diffuse (nuclear expression=cytoplasmic expression) and cytoplasmic (nuclear expression<cytoplasmic expression). In the current study, only YAP distribution localized to the nucleus (nuclear expression $>$ cytoplasmic expression) was deemed as 'nuclear YAP localization'; the 'diffuse' (nuclear and cytoplasmic expression) and 'cytoplasmic' (nuclear<cytoplasmic expression) cells were not included in the criteria.

Reverse transcription-quantitative polymerase chain reaction $(R T-q P C R)$. RT-qPCR was used to analyze the transcriptional levels of Survivin, connective tissue growth factor (CTGF), amphiregulin (AREG) and Ki67. RT-qPCR was performed using an Mx3000P qPCR system (Agilent Technologies, Santa Clara, CA, USA) with SYBR ${ }^{\circledR}$ Premix Ex Taq ${ }^{\mathrm{TM}}$ II (Takara Bio Inc., Shiga, Japan). $\beta$-actin was used as the internal control gene to normalize the levels of target gene expression. Thermocycling conditions were applied as follows: $95^{\circ} \mathrm{C}$ for 
A

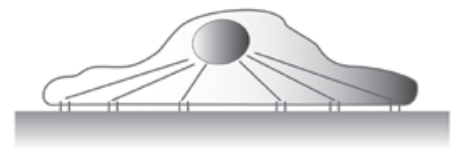

B

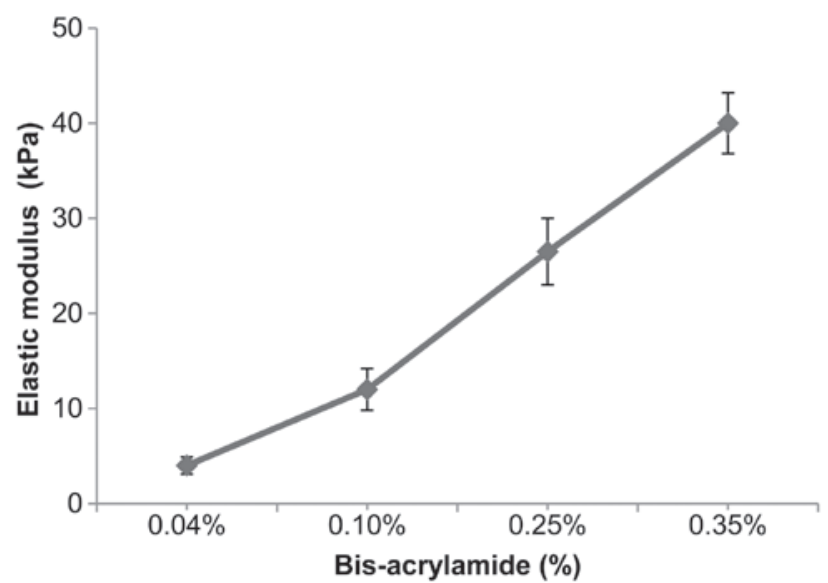

Figure 1. Generation of substrates with varying rigidities. (A) A schematic of the response of non-small cell lung cancer cells to substrates of two different rigidities. (B) Elastic modulus of polyacrylamide gels using 10\% acrylamide and varying bis-acrylamide concentrations.

A

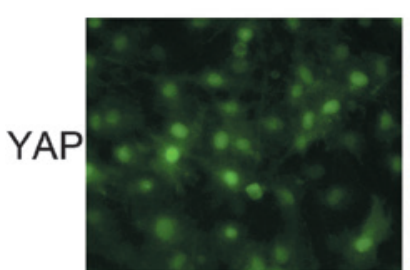

$40 \mathrm{kPa}$

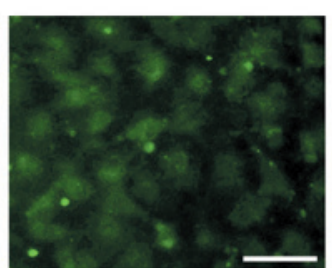

$4 \mathrm{kPa}$
B

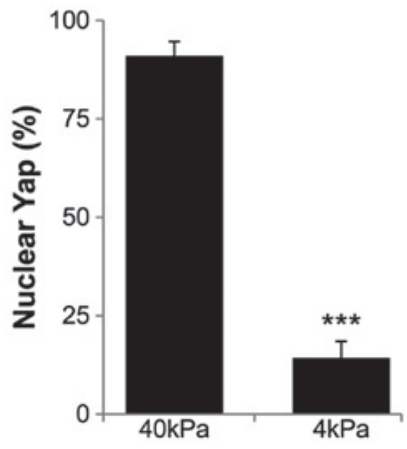

C

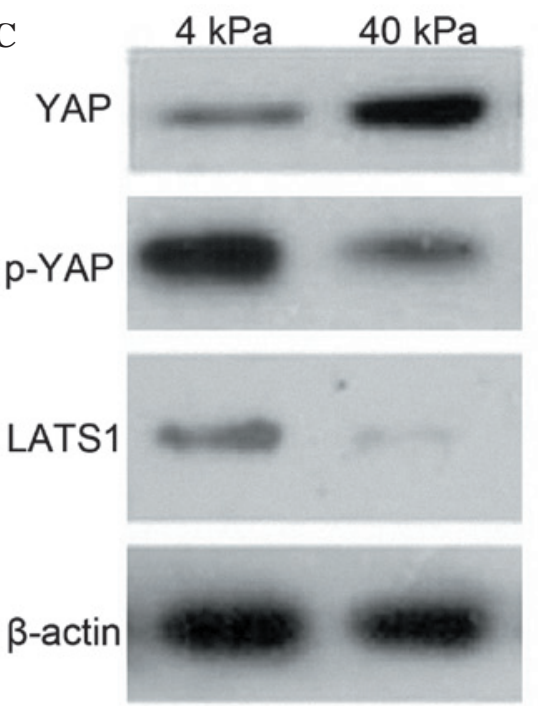

Figure 2. Changes in YAP localization and chondrocyte phenotype on substrates with different rigidities. (A) Immunofluorescence images of YAP (green) in NSCLC cells seeded on substrates with different rigidity. Scale bar, $50 \mu \mathrm{m}$. (B) Graph indicating the percentage of cells with nuclear YAP observed in NSCLC cells on substrates with different rigidities $(\mathrm{n}=5)$. (C) Protein expression levels of YAP, $\mathrm{p}$-YAP and LATS1 in NSCLC cells after two days of culture on a soft substrate $(4 \mathrm{kPa})$ and a stiff substrate $(40 \mathrm{kPa})$. The values represent the mean \pm standard deviation. ${ }^{* * *} \mathrm{P}<0.001$ compared with $40 \mathrm{kPa}$. YAP, Yes-associated protein; NSCLC, non-small cell lung cancer; p-YAP, phospho-YAP; LATS1, large tumor suppressor kinase 1.

$30 \mathrm{sec}, 40$ cycles of denaturation $\left(95^{\circ} \mathrm{C}\right.$ for $\left.5 \mathrm{sec}\right)$, annealing $\left(60^{\circ} \mathrm{C}\right.$ for $\left.30 \mathrm{sec}\right)$ and extension $\left(72^{\circ} \mathrm{C}\right.$ for $\left.30 \mathrm{sec}\right)$. The primers used for the RT-qPCR assays are listed in Table 1. The data were analyzed using the $2^{-\Delta \Delta \mathrm{Ct}}$ method.

Western blot analysis. Cells were lysed in lysis buffer containing protease and phosphatase inhibitors (Sigma-Aldrich). Proteins were separated by sodium dodecyl sulfate-polyacrylamide gel electrophoresis, electroblotted onto polyvinylidene fluoride membranes (Sigma-Alrdich), blocked with 5\% dry milk in Tris-buffered saline with Tween-20, immunoblotted with specific primary antibodies and horseradish peroxidase-conjugated secondary antibodies and detected by enhanced chemiluminescence (Pierce Chemical Co., Rockford, IL, USA). The following rabbit polyclonal IgG antibodies were used (all from Santa Cruz Biotechnology, Inc. unless otherwise stated): Anti-YAP (sc-15407), anti-p-YAP (\#13008; Cell Signaling Technology, Inc., Danvers, MA, USA), anti-LATS (sc-28223), anti-CTGF (sc-25440), anti-AREG (sc-25436) anti-Survivin (sc-10811), and anti-Ki67 (sc-15402), and anti- $\beta$-actin (ab1801; Abcam, Cambridge, MA, USA). The secondary antibody used was the goat anti-rabbit horseradish peroxidase-conjugated IgG H\&L (ab97051; Abcam).

Statistical analysis. At least three replicates were performed in each experiment. Comparison between pairs of groups was performed using the two-samples t-test. SPSS version 13.0 (SPSS, Inc., Chicago, IL, USA) software was used for statistical analysis. $\mathrm{P}<0.05$ was considered to indicate a statistically significant difference. 


\section{Results}

Matrix elasticity increases with an increase in bis-acrylamide. To control the substrate stiffness, fibronectin-coated PA gels with various degrees of stiffness were generated by adjusting the relative quantities of acrylamide and bis-acrylamide crosslinker. When maintaining acrylamide at a constant concentration of $10 \%$, the PA gels with $0.04,0.10,0.25$ and $0.35 \%$ bis-acrylamide ratios yielded elasticities of $4 \pm 0.9,12 \pm 2.2,26.5 \pm 3.5$ and $40 \pm 3.2 \mathrm{kPa}$, respectively (mean \pm standard deviation; Fig. 1B).

Substrate stiffness correlates with YAP and LATS1 kinase expression in NSCLC cells. The SPCA-1 human NSCLC cells were seeded on soft $(4 \mathrm{kPa})$ or stiff $(40 \mathrm{kPa})$ substrates for up to two days. Immunofluorescent staining showed that YAP expression in SPCA-1 cells was largely localized to the nucleus when seeded on the $40-\mathrm{kPa}$ substrate, but was predominantly cytoplasmic on the 4-kPa substrate (Fig. 2A). The percentage of cells with nuclear YAP expression was significantly lower on the 4-kPa substrate than the 40-kPa substrate (Fig. 2B). In addition, expression of the YAP upstream regulators in the Hippo pathway in response to changing substrate stiffness was investigated. The protein expression levels of YAP, p-YAP and LATS1 kinase in SPCA-1 human NSCLC cells in response to substrate stiffness, were analyzed by western blotting following two days of culturing (Fig. 2C). The results showed that levels of LATS1 kinase and p-YAP were extremely low in cells on the stiff substrate $(40 \mathrm{kPa})$ but were markedly increased in cells on the soft substrate $(4 \mathrm{kPa})$. Thus, expression of the YAP protein significantly increased after culturing on the stiff substrate, whereas expression of p-YAP and LATS1 protein markedly decreased after culturing on the stiff substrate (Fig. 2C).

Higher levels of YAP expression and culturing on a stiffer substrate lead to increased proliferation of SPCA-1 NSCLC cells. To evaluate the function of YAP in SPCA-1 NSCLC cells, a knockdown of YAP in these cells was performed. Cells were then cultured on substrates of different stiffness. As shown in Fig. 3A, efficient knockdown of YAP endogenous protein was achieved. The proliferation rates of YAP knockdown cells and control cells were calculated after two days of culture by counting. As shown in Fig. 3B, the proliferation rate of the YAP knockdown cells decreased significantly compared with that of the control cells on the stiff substrate. However, there was no statistical difference in the proliferation rate between the YAP knockdown cells and the YAP control cells when cultured on the soft substrate. Moreover, the proliferation rate of SPCA-1 cells expressing YAP was significantly decreased when plated on the soft substrate, compared with cells plated on the stiff substrate. These findings indicate that increasing matrix stiffness promotes SPCA-1 NSCLC cell proliferation, and that the higher levels of YAP expression in cells grown on a stiff substrate, may in part be the cause of this effect.

CTGF, AREG, Survivin and Ki67 expression alters in response to substrate stiffness and the presence of YAP. To further investigate whether YAP expression in NSCLC, in response to substrate stiffness, is correlated with cell proliferation, the mRNA and protein expression levels of CTGF, AREG, Survivin and Ki-67 (cellular proliferation markers) were examined under
A

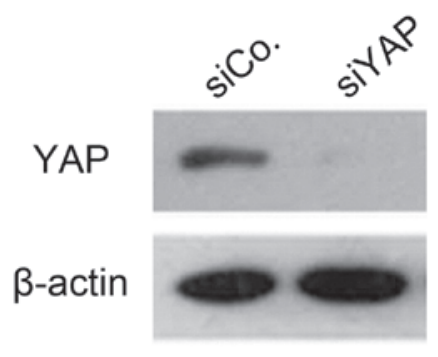

B

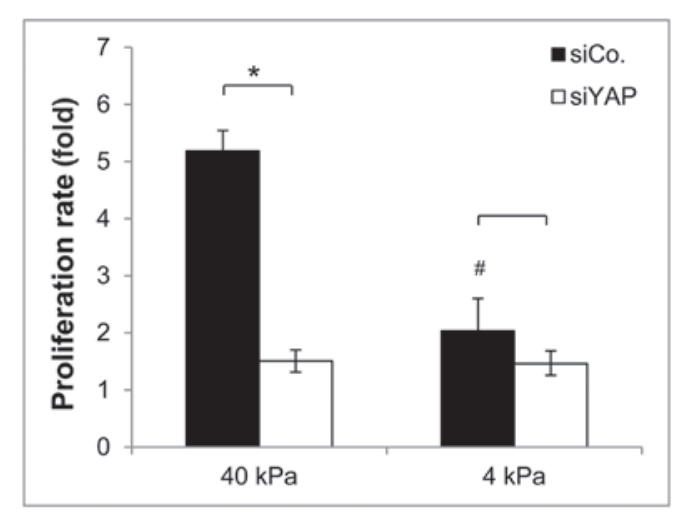

Figure 3. Effects of YAP knockdown in NSCLC cells. (A) Western blotting results show that efficient knockdown of the YAP endogenous protein was achieved using siRNA. (B) The proliferation rate of NSCLC cells cultured for two days under different conditions. Values represent the mean \pm standard error of the mean. ${ }^{*} \mathrm{P}<0.05$, compared with siControl on the $40 \mathrm{kPa}$ substrate. $\# \mathrm{P}<0.05$, cells plated on the $4 \mathrm{kPa}$ substrate compared with cells plated on the $40 \mathrm{kPa}$ substrate. YAP, Yes-associated protein; siCON, small interfering RNA control; siYAP, small interfering YAP RNA.

the different conditions. As shown in Fig. 4, it was found that when culturing on the stiff substrate, the YAP knockdown cells displayed relatively low mRNA expression levels of CTGF, AREG, Survivin and Ki67, compared with the control cells. However, when cells were cultured on the soft substrate, mRNA expression levels of CTGF and Survivin between the knockdown cells and the control cells were not significantly different. For the YAP control cells, the mRNA expression levels of CTGF, AREG, Survivin and Ki67 on the soft substrate were significantly decreased compared with the levels following culture on the stiff substrate.

As shown in Fig. 5, the protein expression levels of CTGF, AREG, Survivin and Ki67 in the YAP knockdown cells were decreased compared with those of control cells on the stiff and soft substrates. Furthermore, protein expression of CTGF, Survivin and Ki67 in SPCA-1 NSCLC cells on the soft substrate also decreased markedly compared with those cultured on the stiff substrate.

\section{Discussion}

The Hippo pathway is an evolutionarily conserved regulator of tissue growth and cell fate (11). The transcription coactivator, YAP, is inhibited by the LATS $1 / 2$ kinases and is a major downstream effector of the Hippo pathway (15). In this study, the role of YAP, and its upstream regulators in the Hippo pathway, in the 
A

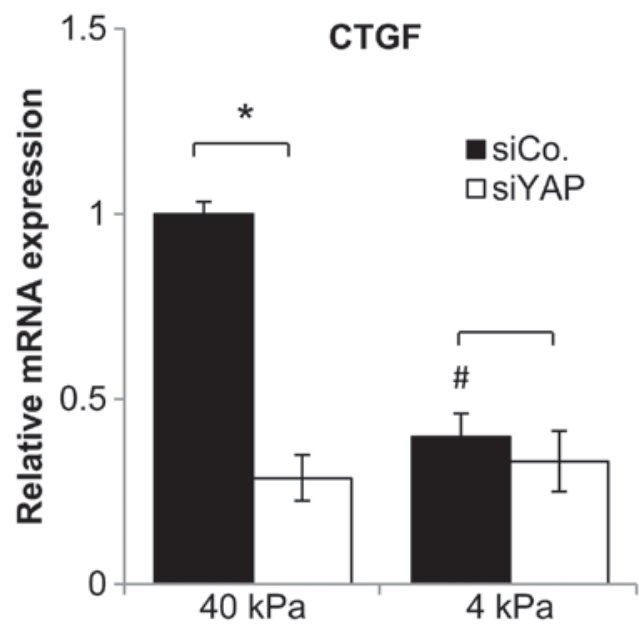

B 1.5 AREG

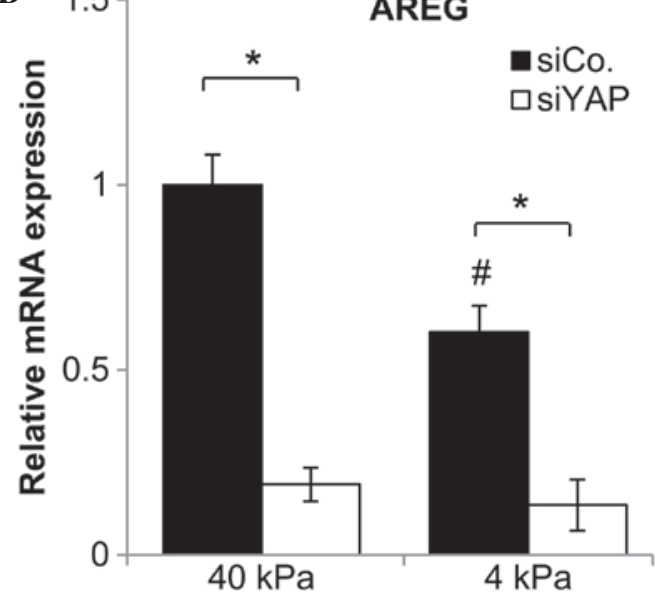

D

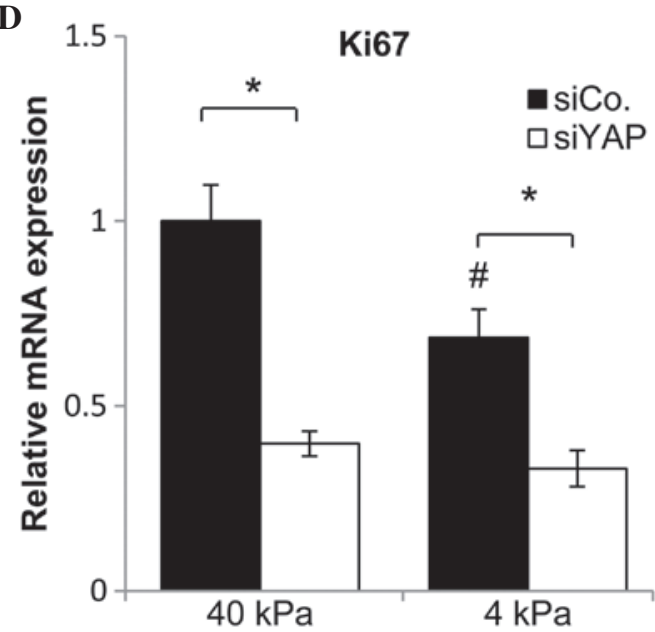

Figure 4. Gene expression levels of (A) CTGF, (B) AREG, (C) Survivin and (D) Ki67 in NSCLC cells, with or without YAP knockdown, after two days of culture on a soft substrate $(4 \mathrm{kPa})$ or a stiff substrate $(40 \mathrm{kPa})$. Data represent the mean \pm standard error of the mean. . $^{*}<0.05$ compared with siControl. $\# \mathrm{P}<0.05$, cells plated on the $4 \mathrm{kPa}$ substrate compared with cells plated on the $40 \mathrm{kPa}$ substrate. CTGF, connective tissue growth factor; AREG, amphiregulin; NSCLC, non-small cell lung cancer; YAP, Yes-associated protein; siCo, small interfering RNA control; siYAP, small interfering YAP RNA.

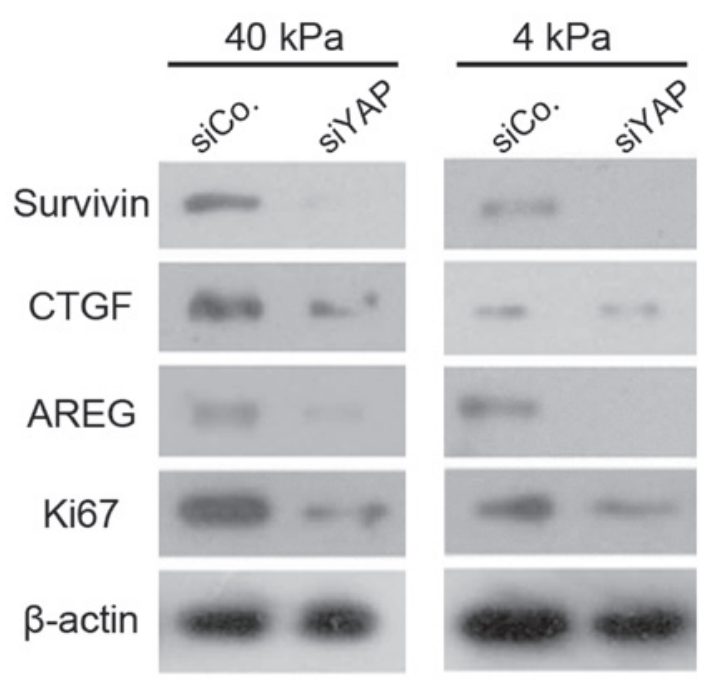

Figure 5. Western blot showing protein expression levels of CTGF, AREG, Survivin and Ki67 in NSCLC cells, with or without YAP knockdown, after two days of culture on a soft substrate $(4 \mathrm{kPa})$ or a stiff substrate $(40 \mathrm{kPa})$. Data represent the mean \pm standard error of the mean. ${ }^{*} \mathrm{P}<0.05$, compared with siControl. CTGF, connective tissue growth factor; AREG, amphiregulin; NSCLC, non-small cell lung cancer, YAP, Yes-accosiated protein SiCon, small interfering RNA control; siYAP, small interfering YAP RNA. regulation of NSCLC cell growth on substrates with different stiffnesses was evaluated. The results support the hypothesis that YAP is a bridging molecule between the growth of SPCA-1 NSCLC cells and the mechanical microenvironment. The findings demonstrate that the stiffness of the subcellular matrix alters the behavior of NSCLC cells in vitro, and that YAP can regulate the growth of NSCLC cells in response to matrix stiffness.

It has previously been demonstrated that mechanical loading can trigger proliferation of tumor cells (16). This implies that signaling pathways that are sensitive to the mechanical properties of their environment may modulate tumor cell behavior. More recently, a study demonstrated that ECM rigidity, cell tension and changes in cell geometry mediate cell contractility, which in turn activates a YAP-dependent mechanoresponse independently of the Hippo cascade (9). It was shown that upon YAP/TAZ depletion, the proliferation rate and the expression levels of cellular proliferation markers in cells plated on a stiff substrate behaved as if they had been plated on a soft substrate. In addition, previous data indicated that the interaction between YAP and TEA domain protein (TEAD) is an integral part of the mechano-tuning mechanism that permits fibroblasts to increase their actomyosin contractility in response to elevated 
ECM stiffness (17). The current study extended these findings to NSCLC cells. It was shown that a hard substrate promoted the growth of NSCLC cells in vitro, as well as an increase in the transcription levels of CTGF, AREG, Survivin and Ki67. This was associated with decreased cytoplasmic expression of YAP and increased nuclear expression, as well as downregulation of p-YAP expression. The results indicated that YAP regulates the proliferation of NSCLC cells in response to the stiffness of the surrounding environment.

Furthermore, CTGF, AREG and Survivin have been identified as potential transcriptional targets of YAP, required for cell growth and differentiation $(18,19)$. Thus, ECM rigidity may influence the expression of CTGF, AREG, Survivin and Ki67 by mediating the expression patterns of YAP, thereby impacting the growth of NSCLC cells. Furthermore, TEAD is also required for YAP-induced cell growth (18), and therefore may also be involved in the stiffness-mediated regulation of NSCLC cell proliferation.

A previous study found that mechanical loading promoted Lewis lung cancer cell growth through induction of periostin expression in the mouse (20). However, whether periostin function correlates with YAP-mediated cell growth in the mechanical microenvironment requires further investigation. In addition, although this study found a positive association between YAP expression and Ki-67 expression (a marker for cell proliferation) in NSCLC cells, the precise mechanisms that are involved in the oncogenic processes of NSCLC development in response to changes in the mechanical microenvironment require further investigation. It is possible that YAP function changes depending on the upstream input, and on variations in the repertoire of binding partners between cells.

This ability of the Hippo pathway to respond to mechanical cues may partially underlie the hyperactivation of YAP and TAZ that has been observed in human tumors, since tumor tissue has altered mechanical properties due to extracellular matrix modifications and the infiltration of stromal and immune cells, which render it more rigid (21). This may also explain why NSCLC tumor tissue is stiffer than the surrounding tissue.

In conclusion, the findings suggest that YAP may be a bridging molecule between NSCLC growth and its mechanical microenvironment, and that it may regulate the growth of NSCLC in response to matrix stiffness. This study provides novel insight into how the growth of NSCLC cells is mediated by physical cues. Modulation of proliferation and apoptosis, controlled by the Hippo-Yap signaling pathway, in lung cancer cells presents an opportunity to develop novel drugs, which may be successfully combined with conventional chemotherapy and radiotherapy treatment strategies.

\section{References}

1. Jemal A, Siegel R, Ward E, et al: Cancer statistics, 2007. CA Cancer J Clin 57: 43-66, 2007.

2. Schiller JH, Harrington D, Belani CP, et al: Comparison of four chemotherapy regimens for advanced non-small-cell lung cancer. New Engl J Med 346: 92-98, 2002.

3. Menon S and Beningo KA: Cancer cell invasion is enhanced by applied mechanical stimulation. PLoS One 6: e17277, 2011.

4. Tse JM, Cheng G, Tyrrell JA, et al: Mechanical compression drives cancer cells toward invasive phenotype. Proc Natl Acad Sci USA 109: 911-916, 2012.

5. Boudreau A, van't Veer LJ and Bissell MJ: An 'elite hacker': breast tumors exploit the normal microenvironment program to instruct their progression and biological diversity. Cell Adh Migr 6: 236-248, 2012.

6. Guilluy C, Swaminathan V, Garcia-Mata R, et al: The Rho GEFs LARG and GEF-H1 regulate the mechanical response to force on integrins. Nat Cell Biol 13: 722-727, 2011.

7. Levental KR, Yu H, Kass L, et al: Matrix crosslinking forces tumor progression by enhancing integrin signaling. Cell 139: 891-906, 2009.

8. Dong J, Feldmann G, Huang J, et al: Elucidation of a universal size-control mechanism in Drosophila and mammals. Cell 130: 1120-1133, 2007.

9. Dupont S, Morsut L, Aragona M, et al: Role of YAP/TAZ in mechanotransduction. Nature 474: 179-183, 2011.

10. Halder G, Dupont S and Piccolo S: Transduction of mechanical and cytoskeletal cues by YAP and TAZ. Nat Rev Mol Cell Biol 13: 591-600, 2012.

11. Harvey KF, Zhang X and Thomas DM: The Hippo pathway and human cancer. Nat Rev Cancer 13: 246-257, 2013.

12. Park JS, Chu JS, Tsou AD, et al: The effect of matrix stiffness on the differentiation of mesenchymal stem cells in response to TGF- $\beta$. Biomaterials 32: 3921-3930, 2011.

13. Tse JR and Engler AJ: Preparation of hydrogel substrates with tunable mechanical properties. Current Protoc Cell Biol, Chapter 10: Unit 10, 16, 2010.

14. Wada K, Itoga K, Okano T, et al: Pathway regulation by cell morphology and stress fibers. Development 138: 3907-3914, 2011.

15. Yu FX, Zhao B, Panupinthu N, et al: Regulation of the Hippo-YAP pathway by G-protein-coupled receptor signaling. Cell 150: 780-791, 2012.

16. Hofmann M, Guschel M, Bernd A, et al: Lowering of tumor interstitial fluid pressure reduces tumor cell proliferation in a xenograft tumor model. Neoplasia 8: 89-95, 2006.

17. Calvo F, Ege N, Grande-Garcia A, et al: Mechanotransduction and YAP-dependent matrix remodelling is required for the generation and maintenance of cancer-associated fibroblasts. Nat Cell Biol 15: 637-646, 2013.

18. Zhao B, Ye X, Yu J, et al: TEAD mediates YAP-dependent gene induction and growth control. Genes Dev 22: 1962-1971, 2008.

19. Huang JM, Nagatomo I, Suzuki E, et al: YAP modifies cancer cell sensitivity to EGFR and survivin inhibitors and is negatively regulated by the non-receptor type protein tyrosine phosphatase 14. Oncogene 32: 2220-2229, 2013.

20. Ma D, Lu H, Xu L, et al: Mechanical loading promotes Lewis lung cancer cell growth through periostin. In Vitro Cell Dev Biol Anim 45: 467-472, 2009.

21. Butcher DT, Alliston T and Weaver VM: A tense situation: forcing tumour progression. Nat Rev Cancer 9: 108-122, 2009. 